\title{
Caracterización de los pacientes con tinnitus y hallazgos audiométricos
}

\section{Characterization of patients with tinnitus and audiometric findings}

\author{
Brian Morales O. ${ }^{1}$, Tatiana Sepúlveda Sch. ${ }^{1}$, Catalina Jury S. ${ }^{1}$, \\ Michelle Bestwick N. ${ }^{1}$, Ricardo Valdés S. ${ }^{1}$, Rodrigo Leiva S. ${ }^{1}$.
}

\section{Resumen}

Introducción: El tinnitus se define como la percepción consciente de un sonido, en ausencia de un estímulo acústico externo correspondiente. Se ha relacionado a múltiples comorbilidades como hipertensión arterial (HTA), diabetes mellitus (DM) e hipoacusia, sin evidencia sólida en la actualidad. Hay diversas formas de experimentar el tinnitus, ya sea de comienzo unilateral o bilateral, intermitente o persistente, con deterioro de la calidad de vida, leve hasta formas invalidantes. El conocer las diversas patologías presentes en el tinnitus, en especial la hipoacusia, toma relevancia al momento de enfrentar a pacientes con dicha patología. Objetivo: Caracterizar el tinnitus y sus principales hallazgos audiométricos en pacientes atendidos en el Servicio de Otorrinolaringología del Hospital Clínico Herminda Martín de Chillán, Chile. Material y Método: Estudio retrospectivo. Se incluyeron pacientes mayores de 18 años con diagnóstico de tinnitus entre los años 2015-2017. Se obtuvo información epidemiológica, comorbilidades, presentación clínica del tinnitus en función de lateralidad y frecuencia, hipoacusia asociada y hallazgos audiométricos. Resultados: Se incluyeron en el estudio a 249 pacientes, la mayoría mujeres $(70 \%)$ con una edad promedio de inicio de su tinnitus entre 60-79 años (52\%). Entre las principales comorbilidades se encuentran la hipertensión arterial, diabetes mellitus y patologías autoinmunes; los síntomas otorrinolaringológicos acompañantes estuvieron en menos del 10\% de los casos. En relación a la caracterización del tinnitus, el 52\% fue de inicio unilateral y el 66\% de forma continua. La hipoacusia objetivada en el audiograma estuvo presente en el $43 \%$ de los pacientes con tinnitus. Conclusión: El tinnitus es una patología compleja y multifactorial, en este sentido, la presencia de hipoacusia objetiva en la audiometría estuvo presente en un $43 \%$ de los pacientes, siendo una de las principales causas a descartar por el médico en el primer enfrentamiento del paciente.

Palabras clave: tinnitus, hipoacusia, audiometría, unilateral, continuo.

\begin{abstract}
Introduction: tinnitus is defined as the conscious perception of a sound, in the absence of a corresponding external acoustic stimulus. It has been related to multiple comorbidities such as high blood pressure, diabetes mellitus, hearing loss, with no solid evidence at present. There are various ways to experience tinnitus, whether it is unilateral or bilateral, intermittent or persistent onset, from mild quality of life deterioration to disabling forms. Knowing the various pathologies present in tinnitus, especially hearing loss, becomes relevant when confronting patients with this pathology. Aim: To characterize tinnitus and its main audiometric findings in patients treated at the Otorhinolaryngology Service of the Herminda Martin of Chillán Clinical Hospital in Chile. Material and Method: Retrospective study. Patients older than 18 years with a diagnosis of tinnitus between the years 2015-2017 were included. Epidemiological information, comorbidities, clinical presentation of tinnitus according to laterality and frequency, associated hearing loss and audiometric findings were obtained. Results: 249 patients were included. Most were women (70\%), with an onset average age of tinnitus between 60 to 79 years (52\%). The accompanying otorhinolaryngological symptoms were in less than 10\% of the cases. The main comorbidities were high blood
\end{abstract}

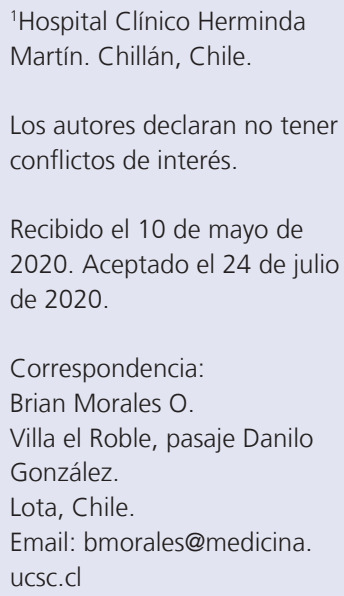


pressure, diabetes mellitus and autoimmune pathologies. 52\% of tinnitus was unilateral presentation and in $66 \%$ it was continuous. $43 \%$ had hypoacusis objectified on the audiogram, regardless of whether they had the sensation of hearing loss. Conclusion: Tinnitus is a complex and multifactorial pathology, in this sense, the presence of objective hearing loss in audiometry was present in $43 \%$ of patients, being one of the main causes to be ruled out by the doctor in the patient's first confrontation.

Keywords: tinnitus, hearing loss, audiometry, unilateral, continuous.

\section{Introducción}

El tinnitus se define como la percepción consciente de un sonido, en ausencia de un estímulo acústico externo correspondiente, comúnmente descrito como percepción fantasma ${ }^{1,2}$, la cual es producto de una actividad bioeléctrica, biomecánica y bioquímica anormal en el oído interno y/o del sistema nervioso central $(\mathrm{SNC})^{2}$. En la literatura, encontramos prevalencias variables en la población general mayor de 50 años, desde valores de 5,1\% hasta $42,7 \%$, principalmente por diferencias a la hora de definir tinnitus ${ }^{3-5}$. Respecto a su presentación, también hay un amplio espectro, desde formas de expresión leves que no conlleven una alteración de la calidad de vida de las personas, hasta condiciones que afectan notablemente el diario vivir, muchas veces en conjunto a otras comorbilidades frecuentes como la depresión, ansiedad e insomnio ${ }^{2,3,6,7}$, incluso últimamente se le ha asociado un rol en el deterioro cognitivo en adultos ${ }^{5,8}$. La disparidad entre ambos polos resalta la naturaleza individual y heterogénea de experimentar el tinnitus.

El tinnitus es una patología que aumenta la prevalencia a mayor edad, donde el peak se alcanza a los 70 años para luego decaer ${ }^{3,6}$ es más frecuente en varones, con gravedad similar entre ambos géneros. El tinnitus puede ser unilateral o bilateral, pulsátil o no pulsátil, intermitente o constante ${ }^{9}$, así como también objetivo o subjetivo, siendo el primero una percepción del sonido que también puede ser escuchada por el examinador, cuya principal patogenia ocurriría debido a un flujo sanguíneo turbulento o contracción muscular, aunque la mayoría de las veces, estemos en presencia de un tinnitus subjetivo, donde el sonido solo lo escucha la persona que lo padece, sin identificarse ninguna fuente de sonido $\mathrm{o}^{6,4,10}$.

El tinnitus se puede experimentar de forma aguda o crónica, es decir, recuperándose espontáneamente en minutos o semanas en la forma aguda o convertirse en un síntoma crónico y debilitante, donde es poco probable que se resuelva de manera espontánea ${ }^{6}$. El tinnitus crónico puede ser episódico con períodos de tiempo asintomático o continuo, donde se caracteriza por episodios mayores de 5 minutos, por al menos 3 meses de duración ${ }^{5,8}$.

El mecanismo fisiopatológico del tinnitus aún no está definido o resuelto, pero existen diversas teorías que tratan de explicarlo, cuyo origen puede encontrarse en cualquier lugar a lo largo de la vía auditiva, desde el oído externo hasta la corteza auditiva ${ }^{9}$. Dentro de sus posibles causas existen muchas, ya sea por disfunciones metabólicas, fármacos, dieta, depresión, patologías de la articulación temporomandibular (ATM), hipertensión arterial, lesiones del SNC y la hipoacusia, donde la transferencia anormal e incontrolada de los impulsos hacia la corteza auditiva podrían ser interpretados como sonidos ${ }^{7}$, encontrándose esta última en el $90 \%$ de los pacientes con tinnitus crónico ${ }^{4}$. Sin embargo, se encuentran otras teorías actuales sobre la génesis del tinnitus en el SNC, donde existe fuerte evidencia de que el tinnitus es una consecuencia directa de respuestas neuroplásticas desadaptativas a la pérdida auditiva, junto a una desactivación sensorial y desinhibición del SNC que permite una hiperactividad espontánea e irregular dentro de las redes neuronales centrales involucradas en el procesamiento del sonido. Junto a lo anterior, se encuentra un aumento de la actividad sincrónica espontánea que ocurre a nivel cortical y subcortical medible mediante electroencefalograma (EEG) y una reorganización funcional, que equivale a un cambio de respuesta de las propiedades de las neuronas dentro de la corteza auditiva primaria a los sonidos externos ${ }^{1,2,6}$. En relación al tratamiento, aunque se han desarrollado una gama de terapias psicológicas, sonoras, eléctricas y electromagnéticas, actualmente no existe una cura confiable para el tinnitus. Al parecer, 
la terapia cognitivo conductual es una de las mejores opciones terapéuticas ${ }^{2,5}$.

La finalidad del estudio, es proporcionar información acerca de las características concomitantes del tinnitus, haciendo especial énfasis en la presencia de hipoacusia objetivada en la audiometría, lo cual pudiera ser relevante para el personal médico a la hora de enfrentar a los pacientes con tinnitus.

\section{Objetivo}

Caracterizar el tinnitus y sus principales hallazgos audiométricos en pacientes atendidos en el Servicio de Otorrinolaringología del Hospital Clínico Herminda Martín de Chillán, Chile.

\section{Material y Método}

Se realizó un estudio retrospectivo, de todos los pacientes mayores de 18 años con el diagnóstico de tinnitus durante los años 2015 a 2017 en el Servicio de Otorrinolaringología del Hospital Clínico Herminda Martín de Chillán. La búsqueda reveló 249 consultas de tinnitus diagnosticadas mediante CIE-10 H93.1. Se revisaron datos epidemiológicos, antecedentes médicos, presentación clínica al inicio del tinnitus (lateralidad y frecuencia), hipoacusia asociada y hallazgos audiométricos en audiograma convencional $(250 \mathrm{a} 8000 \mathrm{~Hz})$, datos que quedaron registrados en la ficha por el especialista. Se excluyeron a pacientes con cirugía otorrinolaringológica de cualquier índole. El estudio cuenta con la aprobación del Comité de Ética del Hospital Clínico Herminda Martín de Chillán.

\section{Resultados}

La serie está constituida por 249 pacientes, con una edad promedio de inicio de los síntomas a los 60 años (rango 20-85 años), si desglosamos por rangos de edad en intervalos de 20 años se observan que la mayor cantidad de pacientes se concentran entre los 60 a 79 años con 129 pacientes representando al $52 \%$. La mayoría de los pacientes fueron de sexo femenino con 175 casos representando al 70\%. Respecto a los distintos síntomas otorrinolaringológicos asociados, se encuentra el antecedente de exposición a ruidos, mareos y vértigo de distintos tipos que iniciaron en conjunto con el tinnitus. Trece pacientes presentaron otalgia ipsilateral al comienzo de su tinnitus. Nueve pacientes refieren un comienzo marcado de su tinnitus como consecuencia de un trauma, traumatismo encefalocraneano, accidente cerebrovascular o posterior a cirugía de bypass coronario. Dos pacientes tenían asociado al comienzo de su tinnitus dolor cervical y tres pacientes con disfunción de la articulación temporomandibular (Tabla 1).

En relación a las formas de presentación del tinnitus, el 37\% de los pacientes se presentó de forma bilateral, el 52\% de forma unilateral, siendo el oído izquierdo el más frecuente con un $65 \%$ de los casos unilaterales. En el 11\% restante no se especificaba la forma de presentación del tinnitus (Figura 1).

En relación a la frecuencia de presentación, el $66 \%$ de los pacientes se presentó de forma continua y el $34 \%$ de forma intermitente o episódica. En 22 pacientes se describe un patrón pulsátil de su tinnitus. En relación a las distintas comorbilidades de los pacientes, la que estuvo presente con mayor frecuencia fue la hipertensión arterial en 127 casos represen-

\begin{tabular}{lcr|}
\hline \multicolumn{3}{c|}{ Tabla 1. Características de pacientes con tinnitus } \\
\hline & $\mathbf{n}$ & $\%$ \\
& pacientes & \\
Edad inicio & & \\
$20-39$ & 15 & 6 \\
$40-59$ & 95 & 38 \\
$60-79$ & 129 & 52 \\
$80+$ & 10 & 4 \\
Total pacientes & 249 & 100 \\
Sexo & & \\
Masculino & 74 & 30 \\
Femenino & 175 & 70 \\
Síntomas otorrinolaringológicos asociados & \\
Vértigo & 32 & 13 \\
Mareos & 12 & 5 \\
Exposición a ruido & 13 & 5 \\
Otalgia ipsilateral & 9 & 4 \\
Prurito ipsilateral & 4 & 2 \\
& & \\
\hline
\end{tabular}




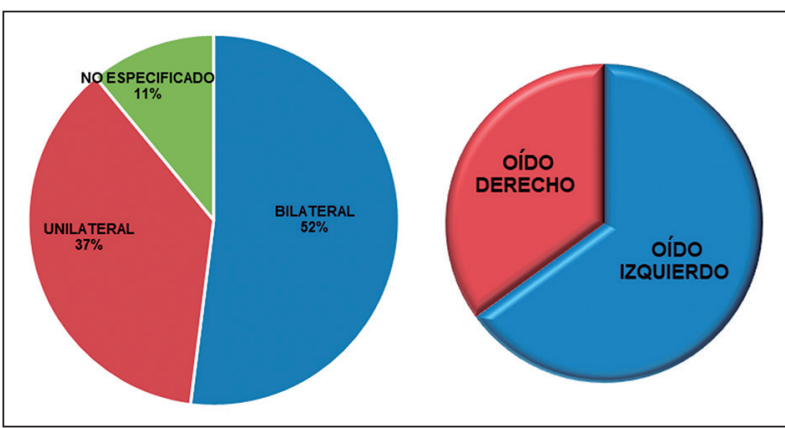

Figura 1. Ubicación del tinnitus. A la izquierda se muestra las principales ubicaciones al inicio del tinnitus. A la derecha se muestra la lateralidad (oído derecho o izquierdo) de los tinnitus unilaterales.

tando el 51\% de la totalidad de los pacientes con tinnitus, le sigue diabetes mellitus con $16 \%$ $(\mathrm{n}=40)$, luego patologías autoinmunes con el $11 \%(\mathrm{n}=28)$ donde se incluyeron patologías tiroideas $(n=20)$, artritis reumatoide $(n=8)$ y lupus eritematoso sistémico $(n=3)$, en tres pacientes coexistían dos patologías autoinmunes. La dislipidemia alcanzó un 9\% $(\mathrm{n}=22)$, el diagnóstico de depresión estuvo presente en 16 pacientes lo que representa el $6 \%(n=16)$, cinco pacientes estaban en tratamiento crónico de cefalea y dos presentaban discopatía cervical. En el estudio no hubo pacientes con diagnóstico ni tratamiento de ansiedad e insomnio (Figura 2).
Es importante aclarar, que se registraron los datos de pacientes con sensación de hipoacusia y con hipoacusia objetiva. Los primeros, fueron pacientes que referían sentir hipoacusia unilateral o bilateral en concomitancia al inicio de su tinnitus, independiente de los resultados de su audiometría y la hipoacusia objetiva fue definida como una audiometría de transmisión, neurosensorial o mixta, con una pérdida mayor o igual a 20 decibeles $^{11}$. El 33\% de los pacientes refiere sentir hipoacusia concomitante a su tinnitus. Al revisar sus audiogramas respectivos, la mayoría presentaba hipoacusia en el audiograma, siendo normal en casi en un tercio de los pacientes, y en cinco pacientes no se describía audiograma, debido a que no fue solicitada o se encontraba en lista de espera para su realización. De los pacientes donde se objetivaba una hipoacusia en el audiograma, en el 63\% la hipoacusia era ipsilateral a la ubicación del tinnitus, en el 27\% la ubicación del tinnitus no es correlacionada con la ubicación de la hipoacusia en el audiograma, es decir, la hipoacusia fue contralateral a la ubicación del tinnitus o bilateral, en cinco pacientes no se describía la ubicación del tinnitus.

Hubo 167 pacientes que no presentaron hipoacusia concomitante con el inicio de su tinnitus. Al revisar sus audiogramas, se observan que en 56 de ellos (34\%) sí había hipoacusia, en 86 pacientes (51\%) la audio-
Figura 2. Comorbilidades en los pacientes con tinnitus. Patologías autoinmunes: hipotiroidismo/hipertiroidismo $(n=20)$, artritis reumatoide $(n=8)$, lupus eritematoso sistémico $(n=3)$.

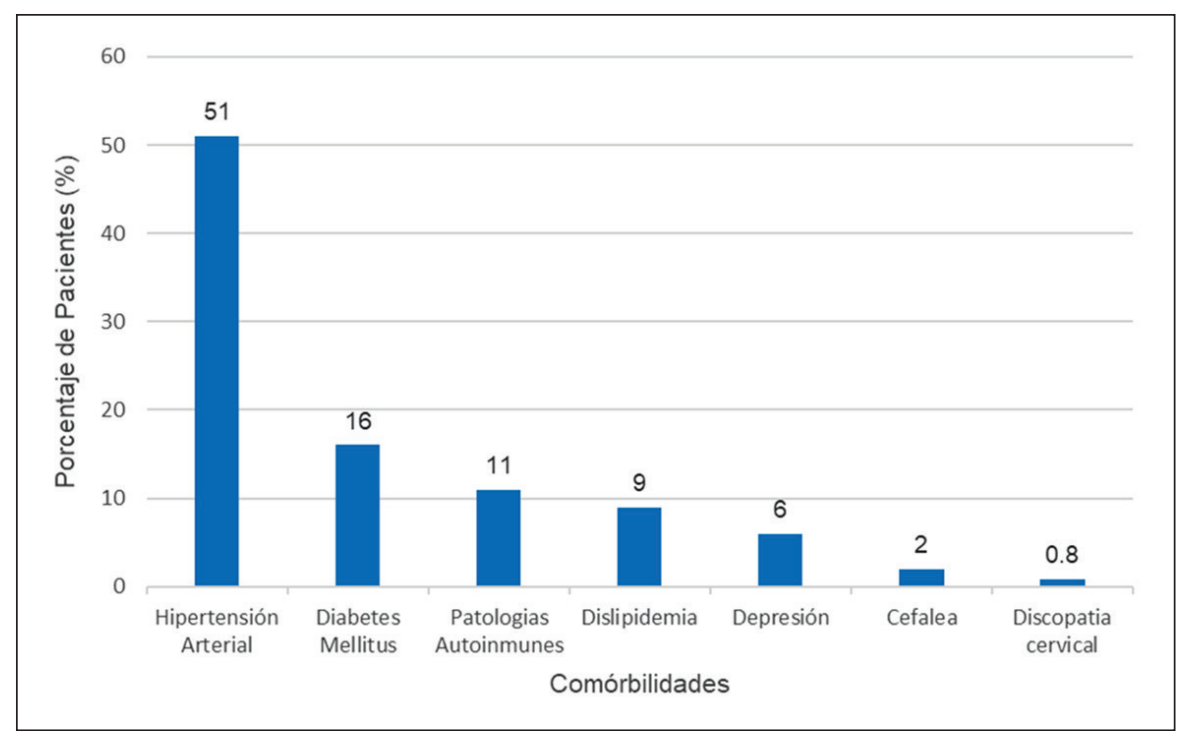




\begin{tabular}{ccc} 
Tabla 2. Distribución de pacientes con sensación de hipoacusia y características de sus & audiogramas \\
\hline & n pacientes & $\%$ \\
Sensación de hipoacusia & 82 & 33 \\
1. Hipoacusia en audiometría (según ubicación) & 51 & 62 \\
- Ipsilateral & 32 & 63 \\
- No ipsilateral & 14 & 27 \\
- No se describe ubicación & 5 & 10 \\
& Total & 100 \\
2. Audiometría normal & 26 & 32 \\
3. Sin audiometría & 5 & 6 \\
Sin sensación de hipoacusia & 167 & 67 \\
1. Hipoacusia en audiometría (según ubicación) & 56 & 34 \\
- Ipsilateral & 31 & 55 \\
- No ipsilateral & 20 & 36 \\
- No se describe ubicación & 5 & 9 \\
& Total & 100 \\
2. Audiometría normal & 86 & 51 \\
3. Sin audiometría & 25 & 15
\end{tabular}

metría resultó normal y en 25 pacientes no se describía la audiometría, por las mismas razones anteriormente mencionadas. De los pacientes donde se objetivaba una hipoacusia en el audiograma, en el 55\% la hipoacusia era ipsilateral a la ubicación del tinnitus, en el 36\% la ubicación de la hipoacusia no se correlacionó con la ubicación del tinnitus, en cinco pacientes no se describía la ubicación del tinnitus. Si tomamos en cuenta a todos los pacientes que tenían hipoacusia objetivada en la audiometría, tengan o no la sensación de hipoacusia, correspondería al $43 \%$ de nuestra muestra total, es decir, en más de la mitad de los pacientes que consultaron por tinnitus no presentaban hipoacusia asociada (Tabla 2).

\section{Discusión}

En nuestro estudio se observa que la aparición del tinnitus aumenta con la edad, alcanzando su mayor frecuencia entre los 60 a 79 años, representando el 52\% del total de pacientes, lo que resulta concordante con lo encontrado en la literatura ${ }^{3,12,13}$, al parecer la edad es un factor desencadenante para la aparición de tinnitus. En el estudio predominó el sexo femenino representando el $70 \%$ de todos los pacientes, esto es distinto a lo encontrado en la literatura donde predomina el género masculino, sin embargo, también se reportan estudios donde el tinnitus afectó en mayor proporción a mujeres o donde la incidencia fue similar entre ambos sexos ${ }^{12,14}$.

En relación a los distintos síntomas otorrinolaringológicos asociados que pudieran ser de mayor interés o donde se establecen posibles relaciones causales, en general su frecuencia fue de baja presentación en nuestro estudio, encontrándose entre el 5\%-10\% de nuestros pacientes, esto contrasta con lo encontrado en la literatura, a modo de ejemplo, en los últimos años se ha relacionado con fuerte evidencia la asociación de patología de la ATM con el desarrollo de tinnitus, donde su prevalencia sería mayor en este grupo ${ }^{15,16}$, pero también se encuentran otros estudios principalmente metaanálisis donde encuentran frecuencias tan variables desde el $9,9 \%$ a $95 \%{ }^{17}$. Una explicación que relacione ambas asociaciones se atribuye a una conexión anatómica, entre la ATM o músculos masticatorios con los músculos del oído medio. Sin embargo, estas amplias variaciones pueden ser atribuidas a diferencias en su metodología, tamaños de muestra, diferencias en el diseño de estudio, 
etc. En el presente estudio, solo tres pacientes presentaban como antecedente patología de ATM.

En relación a la localización, un tercio de los pacientes del estudio iniciaron su tinnitus de forma bilateral, aproximadamente la mitad lo inicia de forma unilateral siendo el más frecuente el oído izquierdo, los cuales son datos concordantes con la literatura ${ }^{14}$, sin embargo, no hay una explicación clara de porqué un oído pudiera ser más frecuentemente afectado que el otro. En relación a la frecuencia, en dos tercios de los pacientes, el tinnitus fue continuo y en un tercio intermitente, esto no se relaciona con la literatura donde la mayor frecuencia es en el patrón intermitente con $60 \%{ }^{14}$. Sin embargo, en un estudio del año 2020, llevada a cabo por Mantello y colaboradores en un hospital general de Sao Paulo, Brasil, encontraron un patrón bilateral en el $60 \%$ de los casos y un patrón continuo en el $80 \%$, siendo similares valores en relación al presente estudio, pero debido a que su muestra solo eran 30 pacientes puede no ser representativo ni generalizable a la población ${ }^{11}$. En relación a las comorbilidades del tinnitus, en la literatura se encuentran amplias asociaciones, pero sin un sustento evidente, se cree que su causa es multifactorial. En el presente estudio la principal comorbilidad fue la hipertensión arterial estando presente en la mitad de los pacientes, sin embargo, en la literatura la evidencia es controvertida ${ }^{18}$. Nuestros resultados podrían explicarse debido a que la HTA es una comorbilidad frecuente en nuestra población, muchas veces en concomitancia a otras patologías cardiovasculares. Una explicación similar sería para la diabetes mellitus, que estuvo presente en un $16 \%$, cuya prevalencia en relación a tinnitus es variable, se postula que la DM a mayor severidad y mayor tiempo de evolución se asocia a mayor riesgo de hipoacusia $y$, por ende, mayor riesgo de tinnitus $^{19}$. La comorbilidad entre tinnitus y trastornos psiquiátricos (depresión, ansiedad, insomnio) es sólida en la literatura, en algunos estudios alcanzan hasta el $45 \%$ y se ha postulado una superposición de redes neuronales y áreas del cerebro involucradas en ambas patologías ${ }^{10,20-22}$. En nuestro estudio la depresión diagnosticada y manejada por neurólogo estuvo presente en $6 \%$ de los pacientes, no tuvimos pacientes con diagnóstico de ansiedad o insomnio. Esto claramente es distinto a lo encontrado en la literatura, quizás un factor confundente y limitación de este estudio fue no estratificar por grados de tinnitus, no sabemos hasta qué punto el tinnitus afectaba la calidad de vida de los pacientes, ya que en los diversos estudios a mayor severidad del tinnitus, mayor frecuencia de depresión, ansiedad o insomnio.

Se ha asociado muchas veces, la relación existente entre la hipoacusia y el tinnitus, lo cual ha sido discutido en muchos trabajos de investigación donde algunos afirman la relación y en otros no. La principal dificultad de esto es que en ambas entidades su prevalencia aumenta con la edad, por lo que establecer causalidad es complejo, muchos pacientes sin sensación de hipoacusia si presentan tinnitus o al revés, pacientes con hipoacusia que nunca desarrollan tinnitus, por lo que aún se desconoce si la pérdida auditiva puede conducir a la aparición de tinnitus ${ }^{8}$. En la literatura, existe una gran asociación entre la presencia de hipoacusia en concomitancia al tinnitus, alcanzando valores que van desde el $84 \%$ a $97 \%{ }^{23,24}$, a raíz de lo cual surge la correlación existente entre la generación del tinnitus y daño auditivo ${ }^{25}$. Sin embargo, en el presente artículo solo un tercio de los pacientes refirió la sensación de hipoacusia concomitante, donde se pone de manifiesto lo heterogéneo y la multicausalidad que hay detrás del tinnitus. $\mathrm{Al}$ revisar las audiometrías de estos pacientes, dos tercios sí presentaba una hipoacusia objetivable en el examen (con una pérdida de más de 20 $\mathrm{dB}^{9}$ ), un tercio de estos pacientes tenían un audiograma normal, es decir, solo presentan tinnitus sin asociación de hipoacusia objetivada. Ahora bien, de los pacientes que presentaban hipoacusia objetivada en audiograma, en dos tercios de ellos, la hipoacusia fue del mismo lado que la presentación del tinnitus. Ahora, de los pacientes que no presentaron la sensación de hipoacusia asociada, al revisar sus audiogramas un tercio de los pacientes si presentó hipoacusia en la audiometría y de estos, en la mitad fue ipsilateral al tinnitus. En este grupo, el 51\% tenían audiometría normal, lo que contrasta un poco con la literatura, donde encuentran un $20 \%$ de normalidad en las audiometrías de pacientes con tinnitus ${ }^{24}$.

En otras palabras, de los pacientes que sí presentan la sensación de hipoacusia, nos 
encontraremos con más de la mitad de los pacientes que tendrán patología detectable por audiometría, y de los pacientes sin la sensación de hipoacusia, un tercio de ellos sí la tendrá. Si lo vemos de un punto de vista global, un 43\% de nuestros pacientes sí presentaron hipoacusia medible en las audiometrías, es decir, es un porcentaje para nada despreciable que involucra a una gran cantidad de pacientes, por lo que a todo paciente que presente tinnitus se le debiera realizar una audiometría y/o ser derivado a un servicio que cuente con tal procedimiento.

\section{Conclusión}

El tinnitus es una patología compleja, multifactorial, con un desarrollo progresivo en investigación y nuevas hipótesis. Nuestro estudio tenía como finalidad conocer las distintas patologías presentes en el tinnitus, su forma de presentación y la presencia o no de hipoacusia en el audiograma. Un 57\% de los pacientes no presentaba hipoacusia objetivada en su audiometría, siendo esta una de las principales causas a descartar por el médico en el primer enfrentamiento del paciente. Se necesitan más estudios, sobre todo del ámbito nacional para establecer la real contribución de la hipoacusia en el desarrollo de tinnitus o si solo es una comorbilidad más asociada.

\section{Bibliografía}

1. Haider H, Hoare D, Costa R, et al. Pathophysiology, Diagnosis and Treatment of Somtosensory Tinnitus: A Scoping Review. Frontiers in Neuroscience. 2017;11:1-11.

2. Zenner H, Delb W, Herwig B, et al. A Multidisciplinary systematic review of the treatment for chronic idiopathic tinnitus. Eur Arch Otorhinolaryngol. 2016;5:2079-2091.

3. McCormack A, Edmondson-Jones M, Somerset S, Hall D. A systematic review of the reporting of tinnitus prevalence and severity. Hear Res. 2016;337:70-79.

4. Fuller T, Cima R, Langguth B, Mazurek B, Vlaeyen JW, Hoare DJ. Cognitive behavioural therapy for tinnitus. Cochrane Database Syst Rev. 2020;1(1):CD012614. Published 2020 Jan 8. doi:10.1002/14651858.CD012614.pub2.
5. Greenwell K, Sereda M, Coulson N, El Refaie A, Hoare DJ. A systematic review of techniques and effects of self-help interventions for tinnitus: Application of taxonomies from health psychology. Int J Audiol. 2016;55 Suppl 3:S79-S89. doi:10.3109/14 992027.2015.1137363.

6. Wegner I, Hall DA, Smit AL, McFerran D, Stegeman I. Betahistine for tinnitus. Cochrane Database Syst Rev. 2018;12(12):CD013093. doi:10.1002/14651858. CD013093.pub2.

7. Skog C, Fjellner J, Ekberg E, Häggman-Henrikson B. Tinnitus as a comorbidity to temporomandibular disorders-A systematic review. J Oral Rehabil. 2019;46(1):87-99. doi:10.1111/joor.12710.

8. Jafari Z, Kolb BE, Mohajerani MH. Age-related hearing loss and tinnitus, dementia risk, and auditory amplification outcomes. Ageing Res Rev. 2019;56:100963. doi:10.1016/j.arr.2019.100963

9. Chari D, Limb C. Tinnitus. Medical Clinics. 2018;102:1081-1093.

10. Chemali Z, Nehmé R, Fricchione G. Sensory neurologic disorders: Tinnitus. Handb Clin Neurol. 2019;165:365-381. doi:10.1016/B978-0-444-640123.00022-8.

11. Mantello E, Lupoli L, Rodrigues P, Cavalcante J, Massuda E, Anastasio A. Functional impact of tinnitus in patients with hearing loss. Int Arch Otorhinolaryngol. 2020; 24:191-197.

12. Oiticica J, Bittar RS. Tinnitus prevalence in the city of São Paulo. Braz J Otorhinolaryngol. 2015;81(2):167176. doi:10.1016/j.bjorl.2014.12.004

13. Bauer CA. Tinnitus. N Engl J Med. 2018;378(13):1224-1231. doi:10.1056/ NEJMcp1506631

14. Curet C, Roitman D. Tinnitus, evaluación y manejo. Rev Med Clin Condes 2016;27:848-862.

15. Song H, Shin J, Lee J, et al. Association between temporomandibular disorders, chronic diseases, and ophthalmologic and otolarynggologic disorders in korean adults: A cross-sectional study. Plos One. 2018; 13:1-16.

16. Algieri GMA, Leonardi A, Arangio P, Vellone V, Paolo CD, Cascone P. Tinnitus in Temporomandibular Joint Disorders: Is it a Specific Somatosensory Tinnitus Subtype? Int Tinnitus J. 2017;20(2):83-87. doi:10.5935/0946-5448.20160016

17. Omidvar S, Jafari Z. Association Between Tinnitus and Temporomandibular Disorders: A Systematic Review and Meta-Analysis. Ann Otol Rhinol Laryngol. 2019;128(7):662-675. doi:10.1177/0003489419842577.

18. Figueiredo RR, de Azevedo AA, Penido Nde O. Tinnitus and arterial hypertension: a systematic review. Eur Arch Otorhinolaryngol. 2015;272(11):3089-3094. doi:10.1007/s00405-014$3277-y$.

19. Eggermont J. Epidemiology and genetics of hearing 
loss and tinnitus. In Hearing Loss. Calgary, Canadá: Elsevier Inc, 2017;209-234.

20. Pattyn T, Van Den Eede F, Vanneste S, et al. Tinnitus and anxiety disorders: a review. Hear Res. 2016; 333:255-265.

21. Aazh H, Lammaing K, Moore BCJ. Factors related to tinnitus and hyperacusis handicap in older people. Int J Audiol. 2017;56(9):677-684. doi:10.1080/14992027.2 017.1335887.

22. Durai M, Searchfield G. Anxiety and depression, personality traits relevant to tinnitus: A scoping review. Int J Audiol. 2016;55(11):605-615. doi:10.1080 /14992027.2016.1198966

23. Manche SK, Madhavi J, Meganadh KR, Jyothy A. Association of tinnitus and hearing loss in otological disorders: a decade-long epidemiological study in a South Indian population. Braz J Otorhinolaryngol.
2016;82(6):643-649. doi:10.1016/j.bjorl.2015.11.007.

24. Tang D, Li H, Chen L. Advances in understanding, diagnosis, and treatment of tinnitus. Adv Exp Biol. 2019; 1130:109-128.

25. Theodoroff SM, Lewis MS, Folmer RL, Henry JA, Carlson KF. Hearing impairment and tinnitus: prevalence, risk factors, and outcomes in US service members and veterans deployed to the Iraq and Afghanistan wars. Epidemiol Rev. 2015;37:71-85. doi:10.1093/epirev/mxu005.

26. Yang P, Ma W, Zheng Y, Yang H, Lin H. A Systematic Review and Meta-Analysis on the Association between Hypertension and Tinnitus. Int J Hypertens. 2015;2015:583493. doi:10.1155/2015/583493.

27. Pattyn T, Van Den Eede F, Vanneste S, et al. Tinnitus and anxiety disorders: a review. Hear Res. 2016; 333:255-265. 